\title{
Urinary incontinence and quality of life: a systematic review and meta-analysis
}

\author{
Damiano Pizzol ${ }^{1}$. Jacopo Demurtas ${ }^{2,3} \cdot$ Stefano Celotto $^{4} \cdot$ Stefania Maggi $^{5} \cdot$ Lee Smith $^{6} \cdot$ Gabriele Angiolelli $^{7}$. \\ Mike Trott $^{6} \cdot$ Lin Yang $^{8,9} \cdot$ Nicola Veronese ${ }^{7,10}$
}

Received: 11 June 2020 / Accepted: 19 August 2020 / Published online: 22 September 2020

(C) The Author(s) 2020

\begin{abstract}
Background Urinary incontinence (UI) and low quality of life (QoL) are two common conditions. Some recent literature proposed that these two entities can be associated. However, no attempt was made to collate this literature. Therefore, the aim of this study was to conduct a systematic review and meta-analysis of existing data to estimate the strength of the association between UI and QoL.

Methods An electronic search of major databases up to 18th April 2020 was carried out. Meta-analysis of cross-sectional and case-control studies comparing mean values in QoL between patients with UI and controls was performed, reporting random-effects standardized mean differences (SMDs) $\pm 95 \%$ confidence intervals (CIs) as the effect size. Heterogeneity was assessed with the $I^{2}$.

Results Out of 8279 articles initially screened, 23 were finally included for a total of 24,983 participants, mainly women. The mean age was $\geq 50$ years in 12/23 studies. UI was significantly associated with poor QoL as assessed by the short-form 36 (SF-36) total score ( $n=6$ studies; UI: 473 vs. 2971 controls; $\mathrm{SMD}=-0.89 ; 95 \% \mathrm{CI}-1.3$ to $\left.-0.42 ; I^{2}=93.5\right)$ and by the sub-scales of SF-36 and 5/8 of the domains included in the SF-36. Similar results were found using other QoL tools. The risk of bias of the studies included was generally high.

Conclusions UI is associated with a poor QoL, with a strong level of certainty. This work, however, mainly based on cross-sectional and case-control studies, highlights the necessity of future longitudinal studies for better understanding the importance of UI on QoL.
\end{abstract}

Keywords Quality of life · Urinary incontinence $\cdot$ Meta-analysis

Electronic supplementary material The online version of this article (https://doi.org/10.1007/s40520-020-01712-y) contains supplementary material, which is available to authorized users.

Nicola Veronese

ilmannato@gmail.com

1 Italian Agency for Development Cooperation, Khartoum, Sudan

2 Clinical and Experimental Medicine PhD Program, University of Modena and Reggio Emilia, Modena, Italy

3 Primary Care Department, USL Toscana Sud Est-Grosseto, Grosseto, Italy

4 Primary Care Department, Azienda Sanitaria Universitaria Friuli Centrale, Udine, Italy

5 National Research Council, Neuroscience Institute, Aging Branch, Padua, Italy
6 Faculty of Sport Sciences, University of Murcia, Murcia, Spain

7 Primary Care Department, Azienda Unità Locale Socio Sanitaria 3 "Serenissima", Venice, Italy

8 Department of Cancer Epidemiology and Prevention Research, Cancer Control Alberta, Alberta Health Services, Calgary, Canada

9 Departments of Oncology and Community Health Sciences, University of Calgary, Calgary, Canada

10 Geriatric Unit, Department of Internal Medicine and Geriatrics, University of Palermo, Palermo, Italy 


\section{Introduction}

Urinary incontinence (UI) assumes an utmost importance in medicine, being a multifactorial syndrome defined as the sign of any involuntary leakage of urine [1-3]. UI is a widespread disorder affecting millions of people over the world with important and probably still underestimated negative consequences on personal and social wellbeing [4]. In particular, UI affects more females than males, even if female UI is yet often underestimated [4]. Although the exact prevalence is not known, at least one person out of four could be affected by UI during their life. [5, 6] UI due to chronic causes can be divided into five groups: urgency, stress, mixed, overflow and functional [7].

Regardless of its type, especially in older adults, UI is rarely reported by the patient, because it is considered a natural consequence of ageing and, most of all, due to a sense of shame [8]. Often, affected individuals deny and hide UI, which results in physical and psychosocial restrictions to enjoyment in life. Actually, the key consequences include loss of self-confidence and social isolation in addition to other negative outcomes such as anxiety, depression, deterioration in sexual life and decrease in physical activity [9]. All these conditions are associated per se with poor quality of life (QoL), an umbrella term that, nowadays, includes various domains in human life that describes the expectations of an individual or society for a good life [10]. Despite increasing research in medicine indicating the importance of QoL and the high prevalence of UI in older adults, no attempt has yet been made to collate the literature investigating the association between UI and QoL in older adults in the attempt to derive a precise understanding on this topic.

Given this background, the aim of this study was to conduct a systematic review and meta-analysis of existing data to estimate the strength of the association between UI and QoL.

\section{Methods}

This systematic review adhered to the PRISMA [11] and MOOSE [12] statements and followed a structured protocol submitted to PROSPERO (https://www.crd.york.ac.uk/prosp ero/display_record.php?RecordID=181768).

\section{Data sources and literature search strategy}

Two investigators (NV and DP) independently conducted a literature search using MEDLINE/PubMed, Scopus, CINAHL, Embase PsycINFO and Cochrane Library databases from inception until 18th April 2020. Any inconsistencies were resolved by consensus with a third author (JD).

In PubMed, the following search strategy was used: "(urine incontinence OR bladder incontinence OR incontinence, urine OR urinary incontinence OR urinary leakage OR urine bladder incontinence OR urine incontinence OR urine leakage OR wetting, urine) AND (quality of life OR hrql OR health related quality of life OR life quality)". Conference abstracts and reference lists of included articles were hand-searched to identify any potential additional relevant work.

\section{Study selection}

Following the PICOS (participants, intervention, controls, outcomes, study design) criteria, we included studies assessing:

P People with urinary incontinence

I None

C People without urinary incontinence

O Quality of life

S Observational (case-control, cross-sectional, cohort studies)

The diagnosis of UI could be made through selfreported information, through validated generic (e.g., Katz's index [13]) or specific (e.g., Sandvik Severity Index [14]) questionnaires or instrumental tools (e.g., urodynamic tests); QoL was assessed through validated tools that are summarized in Supplementary Table 1. Studies were excluded if they included pediatric populations; if the data were not analyzable; or if they did not clearly report data regarding QoL tools in UI and/or controls. No language restriction was placed.

\section{Data extraction}

For each eligible study, two independent investigators (NV, DP) extracted: name of the first author and year of publication, setting, sample size, mean age of the population, $\%$ of females, ethnicity, prevalence of some comorbidities related to urinary incontinence and QoL (such as $\%$ of dermatitis, $\%$ of disability and others), diagnostic tool used for QoL and for UI definitions, and the severity of the UI. These data were extracted, if possible, for those with UI and for controls, respectively. Data about matching and method (i.e., propensity score) were planned to be extracted, but no study included this information. 


\section{Outcomes}

The primary outcomes were considered the mean values and the correspondent standard deviations (SDs) of the validated tools of QoL, comparing the values of participants with UI and the controls. If the data were reported in other ways, e.g., median and interquartile ranges, they were transformed into means and SD.

\section{Assessment of study quality}

Two independent authors (SC, JD) made the assessment of the studies' quality using the Newcastle-Ottawa Scale (NOS) [15]. The NOS assigns a maximum of 9 points based on three quality parameters: selection, comparability, and outcome. As per the NOS grading in past reviews, we graded studies as having a high ( $<5$ stars), moderate (5-7 stars) or low risk of bias ( $\geq 8$ stars) [16].

\section{Data synthesis and statistical analysis}

All analyses were performed using Stata, version 15.0. For all analyses, a $p$ value less than 0.05 was considered statistically significant.

The primary analysis compared the values of QoL tests between participants with UI vs. controls, according to the test used for assessing the QoL. We calculated the difference between the means of the UI and control groups through standardized mean differences (SMD) with their 95\% confidence intervals (CIs), applying a random-effect model [17]. We then applied the indications for interpreting the magnitude of the SMD in the social sciences, as suggested by Cohen [18], i.e., small, $\mathrm{SMD}=0.2-0.5$; medium, $\mathrm{SMD}=0.5-0.8$; and large, $\mathrm{SMD}>0.8$. The data were also reported as forest plots, in a graphical way.

Heterogeneity across studies was assessed by the $I^{2}$ metric. Given significant heterogeneity $\left(I^{2} \geq 50 \%\right.$ and/or $p<0.05$ ) [19] and having at least 10 studies for each outcome, we planned to run meta-regression analyses, taking as moderators the factors cited in the data extraction paragraph (see for more information Supplementary Table 2) in the sample as whole and as differences, in prevalence, between UI and controls. However, no outcome included 10 studies and so these analyses were not possible. Since the causes of UI are traditionally different between men and women, we assessed the percentage of women in each study as potential moderator of highly heterogeneous findings, having at least four studies for an outcome.

Publication bias was assessed by visual inspection of funnel plots and using the Egger bias test [20]. In case of publication bias, when $\geq 3$ studies were available, we used the Duval and Tweedie non-parametric trim-and-fill method to account for potential publication bias [21]. Based on the assumption that the effect sizes of all the studies are normally distributed around the center of a funnel plot, in the event of asymmetries, this procedure adjusts for the potential effect of unpublished (trimmed) studies [20]. However, no outcome suffered on publication bias.

\section{Results}

\section{Literature search}

As shown in Fig. 1, we initially found 8279 possible eligible articles. After removing 7981 works through the title/ abstract screening, 298 were retrieved as full text. Of the 298 full text, 23 satisfied the inclusion/exclusion criteria and were, then, included in the systematic review and metaanalysis [22-44].

\section{Descriptive data}

The 23 studies included a total of 24,983 participants ( 8723 with UI; 16,260 controls). The mean age was $\geq 50$ years in $12 / 23$ studies and were mainly women (only women: 14 studies; more than 50\% women: 8 studies; only men: 1 study) (Table 1). The studies were mainly cross-sectional (14 cross-sectional and 9 case-control), made in America (8 studies) and mainly included outpatients (19 studies). UI was diagnosed mainly through self-reported information (18 studies). Only a few studies reported details regarding UI: namely 4 studies included only stress UI and 3 studies a mix of stress-urgency-mixed incontinence. Only one study reported the severity of UI (mild, moderate, severe) [29]. QoL was assessed mainly through short-form 36 (SF36) [45] (10 studies), followed by other tools in order of frequency.

\section{Urinary incontinence and quality of life}

As shown in Tables 2 and 3 and graphically in Fig. 2, UI was significantly associated with poor QoL. For example, for the SF-36 (total score), we observed in six studies (UI: 473 vs. 2971 controls $)$ a $\mathrm{SMD}=-0.89(95 \% \mathrm{CI}-1.3$ to -0.42 ; $I^{2}=93.5$ ), indicating a large association between UI and low QoL comparing to controls. The same results presented for SF-36 sub-scales, i.e., for SF-36 mental and for SF-36 physical scores, where the association was medium and large, respectively, in 8 studies including 4604 participants with UI and 10,121 controls.

When assessing the singular domain of the SF-36, UI was associated with significant worse scores in general health perception, physical and social function, mental health and vitality, with a medium-large strength of these associations (Table 2). 
Fig. 1 PRISMA flow-chart

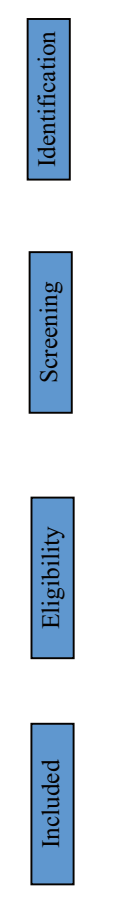

Similar findings were presented when using the Incontinence Impact Questionnaire (IIQ-7) [46] in five studies, including 474 participants with UI and 1279 controls. Using this tool, the SMD was 0.34 (95\% CI $0.01-0.67 ; I^{2}=88 \%$ ) (Table 3, Fig. 2). The percentage of women in the studies was not associated with worse QoL, in meta-regression analysis.

Finally, statistically significant results were found when using other tools for assessing QoL, even if these outcomes included $\leq 3$ studies (Table 3, Fig. 2).

\section{Publication bias}

As fully reported in Table 1, no included outcome suffered on publication bias.

\section{Risk of bias}

The risk of bias, evaluated through the NOS, was fully reported in Table 1 (as total score) and Supplementary Table 3 (for case-control studies) and 4 (for cross-sectional studies), respectively. Six case-control studies over 9 suffered on low quality (high risk of bias) as indicated by a NOS $<5 / 9$. The selection and the representativeness of cases and controls were predominant problems in these studies (Supplementary Table 3).

Furthermore, half of the cross-sectional studies suffered on potential high risk of bias. Again, issues regarding the sample size definition, poor descriptions of non-respondent and lack of matching were the main shortcomings for these studies (Supplementary Table 4).

\section{Discussion}

In this systematic review and meta-analysis, including 23 studies and 24,983 participants ( 8723 with UI; 16,260 controls), we found that the presence of UI was significantly associated with poor QoL. These results, even if characterized by a high heterogeneity and a potential high risk of bias, are of importance, since they add new insight regarding this important topic.

Previously, approximately 10-years ago at the time of writing, two systematic reviews without any formal metaanalysis reached similar conclusions. One review [47] reported that women with UI had lower QoL than their counterparts, but the findings were limited by the small sample size included; the other systematic review [48] found that overactive bladder can be associated with lower QoL levels, but did not include any other UI type. The present metaanalysis overcomes these inherent limitations. First, this review included both men and women. Even if UI is a typical condition of women [49], increasing research is showing the importance of UI in men [50]. Second, all types of UI and not only overactive bladder were included in the present review. Finally, this work incorporated a meta-analytic approach, quantifying the possible association between UI 


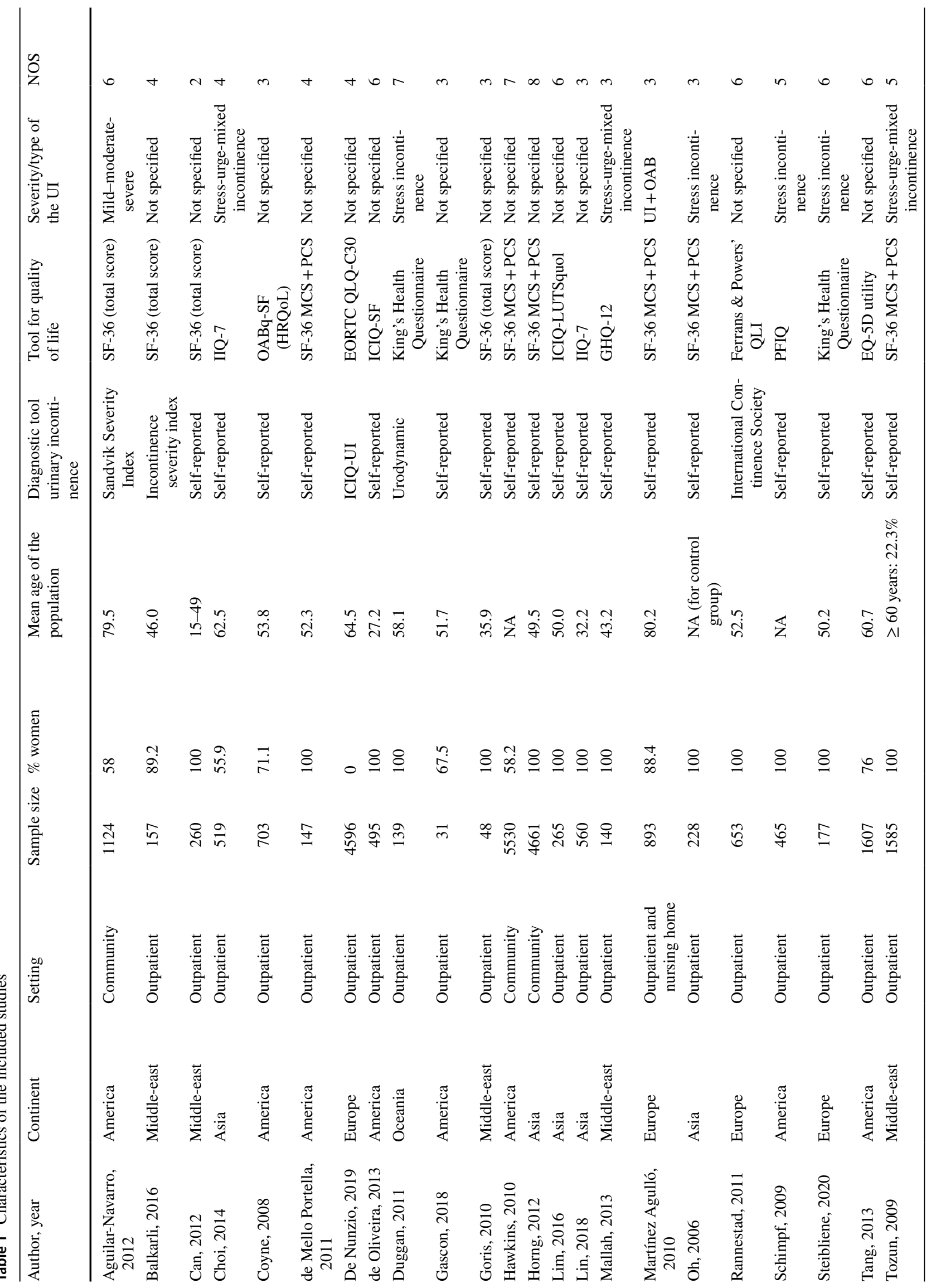




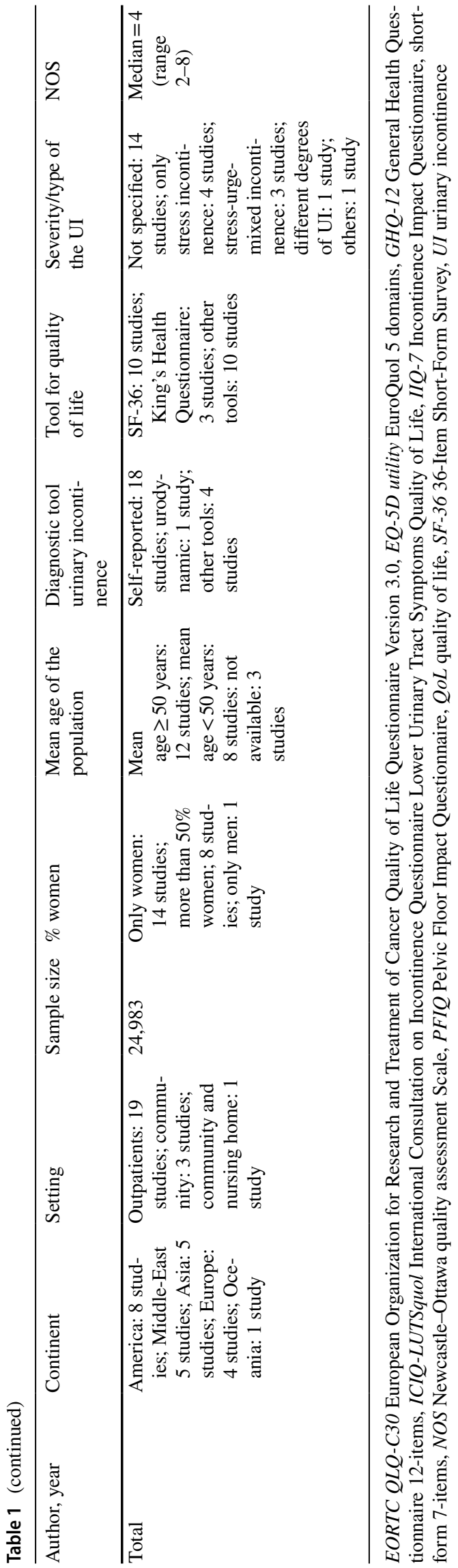

and QoL showing that UI is associated with a poor QoL with a strong/medium strength.

UI may be associated with poor QoL via several mechanisms. First, people with UI usually exhibit more comorbidities than those without. Although several risk factors are reported, the most specifically related are sex, age, dementia, and mobility ability [51]. In addition, fluid intake, selfmobility, diuretic treatment may also influence diuresis and thus UI [51]. It is widely known that all these factors are associated with poor QoL in older people. We have tried to explore the role of comorbidities for explaining our findings, but, unfortunately, no sufficient data are presented in the studies included, as shown in Supplementary Table 2. Second, it is possible that people having UI can use diapers and the use of these tools can lead, in particular conditions, to the Incontinence-Associated Dermatitis (IAD) [52]. IAD, as other dermatological conditions, is associated with a poor QoL [53]: unfortunately, no one of the 23 studies included, reported data regarding this important condition that should be explored in future studies. Finally, we believe that poor QoL in UI can be justified by the presence of shame in these people leading to a change in their lifestyle and habits [54] (i.e., reduction or suppression of physical activity) and to a development of mental disorders (i.e., depression [54] and anxiety [55]). This could be particularly true in younger people [8].

Finally, as reported in a systematic review published over a decade ago [56], only a few interventions are able to improve QoL in people affected by UI. Across 96 randomized controlled trials included, one study including 451 women reported that duloxetine significantly improved QoL compared to placebo [57], whilst pelvic-floor muscle exercise position did not affect QoL in patients affected by UI [58]. These findings suggest that more research is needed for better understanding pharmacological and non-pharmacological interventions able to improve QoL in UI.

The findings of our study should be interpreted within its limitations. First, only case-control and cross-sectional studies were included, and these studies have inherent limitations, potentially introducing a reverse bias (i.e., people with poor QoL for other reasons may experience UI). Second, the included studies mainly encompassed women as participants, but UI is an important condition also in men: the results of this study may thereby not be directly applicable to a male population. Third, the results were highly heterogeneous and thus it is not possible to explain this issue through a meta-regression, since the data reported for the moderators and planned in our protocol are too inconclusive. Finally, several studies are at high risk of bias. Of importance, no study preformed matching between participants with UI and controls, potentially introducing a bias.

In conclusion, the present systematic review and metaanalysis showed that UI is associated with a poorer QoL 
Table 2 Main findings regarding quality of life (QoL) and urinary incontinence (UI) using short-form 36

\begin{tabular}{|c|c|c|c|c|c|c|c|c|c|}
\hline Tools for QoL & $\begin{array}{l}\text { Number of } \\
\text { comparisons }\end{array}$ & UI & Controls & SMD & $95 \% \mathrm{CI}$ & & $p$ value & $I^{2}$ & Egger's test ( $p$ value) \\
\hline SF-36 (total score) & 6 & 473 & 2971 & -0.89 & -1.3 & -0.42 & $<0.0001$ & 93.5 & $\begin{array}{l}-10.0 \pm 4.27 \\
(0.06)\end{array}$ \\
\hline SF-36 Mental & 8 & 4604 & 10,121 & -0.52 & -0.75 & -0.29 & $<0.0001$ & 96.9 & $\begin{array}{l}-2.29 \pm 7.08 \\
(0.76)\end{array}$ \\
\hline SF-36 Physical & 8 & 4604 & 10,121 & -1.04 & -1.39 & -0.69 & $<0.0001$ & 98.6 & $\begin{array}{l}-5.17 \pm 3.61 \\
(0.20)\end{array}$ \\
\hline General health perception & 8 & 4604 & 10,121 & -0.76 & -1.00 & -0.52 & $<0.0001$ & 94.8 & $\begin{array}{l}-15.1 \pm 7.27 \\
(0.26)\end{array}$ \\
\hline Physical function & 7 & 4392 & 9835 & -1.04 & -1.46 & -0.63 & $<0.0001$ & 97.9 & $\begin{array}{l}-6.28 \pm 12.27 \\
(0.96)\end{array}$ \\
\hline Social function & 7 & 4392 & 9835 & -0.44 & -0.71 & -0.18 & 0.001 & 95 & $\begin{array}{l}-5.42 \pm 8.45 \\
(0.48)\end{array}$ \\
\hline Phys role function & 7 & 4392 & 9835 & -0.91 & -1.88 & 0.07 & 0.07 & 99.6 & $\begin{array}{l}-12.54 \pm 8.27 \\
(0.46)\end{array}$ \\
\hline Emotional role function & 7 & 4392 & 9835 & 0.07 & -0.02 & 0.34 & 0.61 & 95.1 & $\begin{array}{l}8.21 \pm 8.24 \\
(0.96)\end{array}$ \\
\hline Mental health & 7 & 4392 & 9835 & -0.58 & -0.88 & -0.27 & $<0.0001$ & 96.3 & $\begin{array}{l}-4.56 \pm 4.68 \\
(0.98)\end{array}$ \\
\hline Vitality & 7 & 4392 & 9835 & -0.50 & -0.76 & -0.24 & $<0.0001$ & 94.9 & $\begin{array}{l}-8.45 \pm 8.27 \\
(0.95\end{array}$ \\
\hline Pain & 7 & 4392 & 9835 & -0.21 & -0.52 & 0.10 & 0.19 & 96.4 & $\begin{array}{l}-2.54 \pm 2.27 \\
(0.96)\end{array}$ \\
\hline
\end{tabular}

CI confidence interval, EORTC QLQ-C30 European Organization for Research and Treatment of Cancer Quality of Life Questionnaire Version 3.0, EQ-5D utility EuroQuol 5 domains, GHQ-12 General Health Questionnaire 12-items, ICIQ-LUTSquol International Consultation on Incontinence Questionnaire Lower Urinary Tract Symptoms Quality of Life, IIQ-7 Incontinence Impact Questionnaire, short-form 7-items, PFIQ Pelvic Floor Impact Questionnaire, $Q o L$ quality of life, $S F$-36 36-Item Short-Form Survey, SMD standardised mean difference, $U I$ urinary incontinence

Table 3 Main findings regarding quality of life (QoL) and urinary incontinence (UI) using other tools for assessing QoL

\begin{tabular}{|c|c|c|c|c|c|c|c|c|c|}
\hline Tools for QoL & $\begin{array}{l}\text { Number of } \\
\text { comparisons }\end{array}$ & UI & Controls & SMD & $95 \% \mathrm{CI}$ & & $p$ value & $I^{2}$ & Egger's test ( $p$ value) \\
\hline IIQ-7 & 5 & 474 & 1279 & 0.34 & 0.01 & 0.67 & 0.04 & 88 & $\begin{array}{l}0.07 \pm 7.88 \\
(0.99)\end{array}$ \\
\hline $\begin{array}{l}\text { EORTC } \\
\text { QLQ-C30 }\end{array}$ & 1 & 771 & 3825 & 0.20 & 0.12 & 0.28 & $<0.0001$ & - & - \\
\hline King's Health Questionnaire & 3 & 196 & 151 & 0.23 & -0.17 & 0.62 & 0.26 & 61.5 & $\begin{array}{l}-2.32 \pm 3.60 \\
(0.64)\end{array}$ \\
\hline ICIQ-LUTSquol & 1 & 120 & 145 & 2.52 & 2.20 & 2.85 & $<0.0001$ & - & - \\
\hline GHQ-12 & 3 & 105 & 105 & 0.31 & 0.01 & 0.61 & 0.04 & 16.3 & $\begin{array}{l}107 \pm 26 \\
(0.15)\end{array}$ \\
\hline Ferrans \& Powers' & 1 & 225 & 428 & -6.29 & -6.67 & -5.92 & $<0.0001$ & - & - \\
\hline PFIQ & 3 & 354 & 333 & 1.29 & 0.41 & 2.16 & 0.004 & 96.0 & $\begin{array}{l}7.32 \pm 31.38 \\
(0.85)\end{array}$ \\
\hline EQ-5D utility & 1 & 907 & 700 & -0.38 & -0.48 & -0.28 & $<0.0001$ & - & - \\
\hline
\end{tabular}

CI confidence interval, EORTC QLQ-C30 European Organization for Research and Treatment of Cancer Quality of Life Questionnaire Version 3.0, EQ-5D utility EuroQuol 5 domains, GHQ-12 General Health Questionnaire 12-items, ICIQ-LUTSquol International Consultation on Incontinence Questionnaire Lower Urinary Tract Symptoms Quality of Life, IIQ-7 Incontinence Impact Questionnaire, short-form 7-items, PFIQ Pelvic Floor Impact Questionnaire, $Q o L$ quality of life, SF-36 36-Item Short-Form Survey, SMD standardised mean difference, UI urinary incontinence 
Fig. 2 Association of urinary incontinence with quality of life, effect sizes represented in standard mean difference (SMD) and 95\% confidence intervals

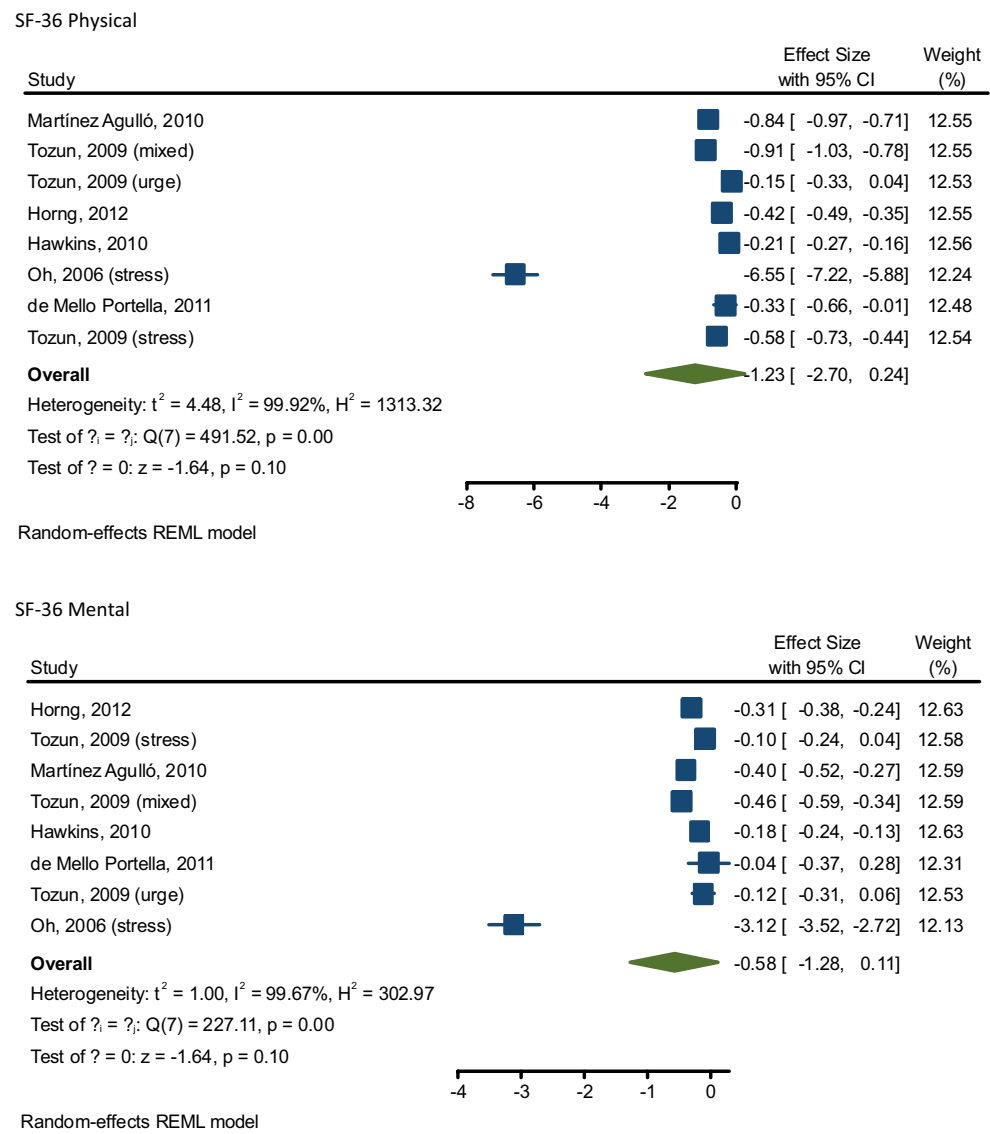

Random-effects REML model

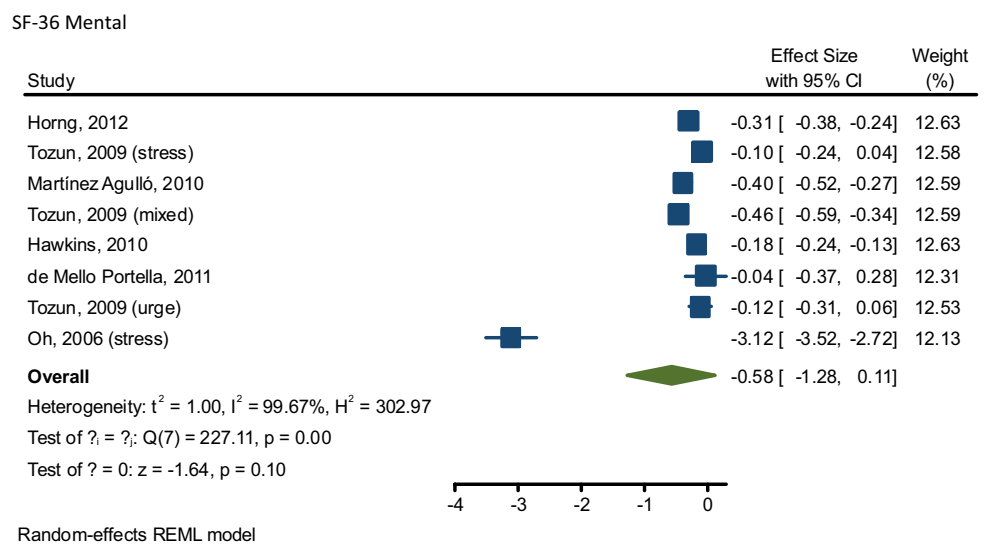

SF-36 (total score)

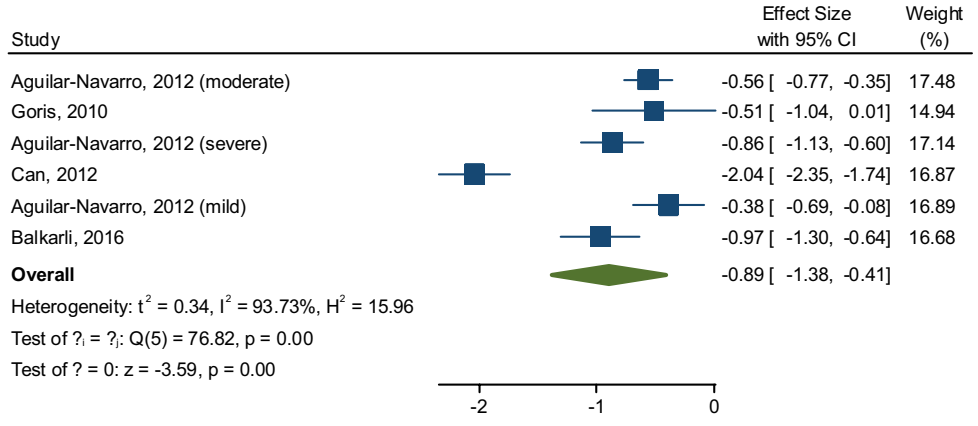

Random-effects REML model

Effect Size Weight with $95 \% \mathrm{Cl} \quad(\%)$

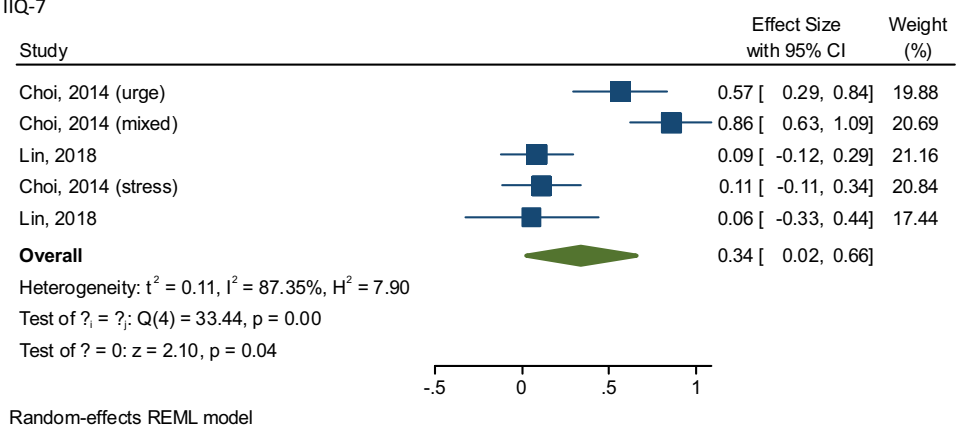


Fig. 2 (continued)

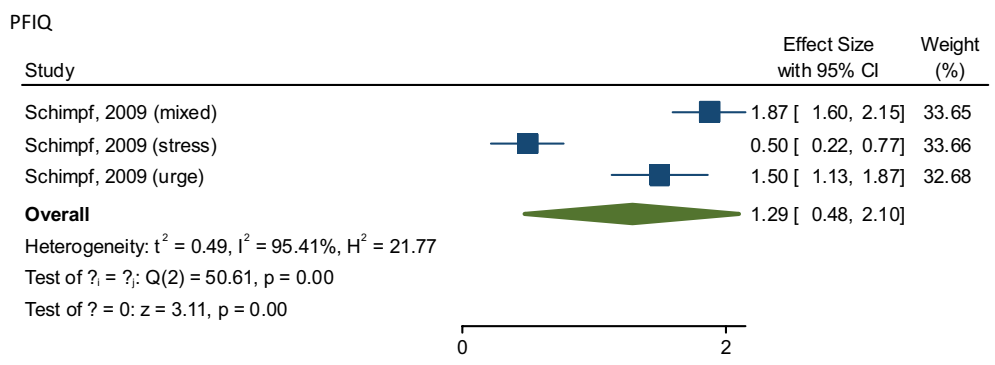

Random-effects REML model

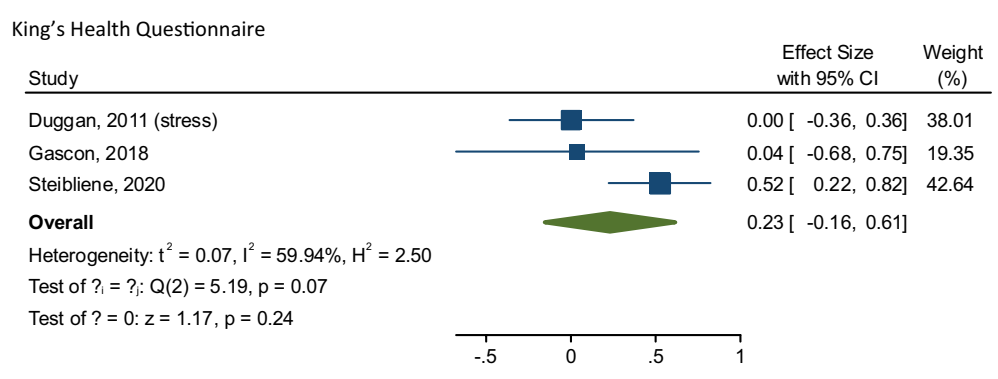

Random-effects REML model

\begin{tabular}{l} 
GHQ - 12 \\
Study \\
\hline Mallah, 2013 (mixed) \\
Mallah, 2013 (urge) \\
Mallah, 2013 (stress) \\
Overall \\
Heterogeneity: $\mathrm{t}^{2}=0.01, \mathrm{I}^{2}=16.40 \%, \mathrm{H}^{2}=1.20$ \\
Test of $?=$ ?:: $\mathrm{Q}(2)=2.39, \mathrm{p}=0.30$ \\
Test of $?=0: \mathrm{z}=2.05, \mathrm{p}=0.04$
\end{tabular}

when compared to controls, with a strong level of certainty. This work, mainly based on cross-sectional and case-control studies at high risk of bias, highlights the necessity of future longitudinal studies for better understanding the importance of UI in determining QoL.

Funding This study was supported by an unrestricted grant of Santex S.P.A.

\section{Compliance with ethical standards}

Conflict of interest The author(s) declare that they have no competing interests.

Open Access This article is licensed under a Creative Commons Attribution 4.0 International License, which permits use, sharing, adaptation, distribution and reproduction in any medium or format, as long as you give appropriate credit to the original author(s) and the source, provide a link to the Creative Commons licence, and indicate if changes were made. The images or other third party material in this article are included in the article's Creative Commons licence, unless indicated otherwise in a credit line to the material. If material is not included in the article's Creative Commons licence and your intended use is not permitted by statutory regulation or exceeds the permitted use, you will need to obtain permission directly from the copyright holder. To view a copy of this licence, visit http://creativecommons.org/licenses/by/4.0/.

\section{References}

1. Aharony L, De Cock J, Nuotio M et al (2017) Consensus document on the management of urinary incontinence in older people. Eur Geriatr Med 8:210-215

2. Abrams P, Cardozo L, Fall M et al (2002) The standardisation of terminology of lower urinary tract function: report from the Standardisation Sub-committee of the International Continence Society. Am J Obstet Gynecol 187:116-126

3. Veronese N, Soysal P, Stubbs B et al (2018) Association between urinary incontinence and frailty: a systematic review and meta-analysis. Eur Ger Med 9:571-578

4. Dooley Y, Kenton K, Cao G et al (2008) Urinary incontinence prevalence: results from the National Health and Nutrition Examination Survey. J Urol 179:656-661 
5. Stewart WF, Hirsh AG, Kirchner HL et al (2014) Urinary incontinence incidence: quantitative meta-analysis of factors that explain variation. J Urol 191:996-1002

6. Ebbesen MH, Hunskaar S, Rortveit G et al (2013) Prevalence, incidence and remission of urinary incontinence in women: longitudinal data from the Norwegian HUNT study (EPINCONT). BMC Urol 13:27

7. Gibbs CF, Johnson TM 2nd, Ouslander JG (2007) Office management of geriatric urinary incontinence. Am J Med 120:211-220

8. Elenskaia K, Haidvogel K, Heidinger C et al (2011) The greatest taboo: urinary incontinence as a source of shame and embarrassment. Wien Klin Wochen 123:607-610

9. Farage MA, Miller KW, Berardesca E et al (2008) Psychosocial and societal burden of incontinence in the aged population: a review. Arch Gynecol Obstet 277:285-290

10. Guyatt GH, Feeny DH, Patrick DL (1993) Measuring healthrelated quality of life. Ann Int Med 118:622-629

11. Liberati A, Altman DG, Tetzlaff J et al (2009) The PRISMA statement for reporting systematic reviews and meta-analyses of studies that evaluate health care interventions: explanation and elaboration. PLoS Med 6:e1000100

12. Stroup DF, Ja Berlin, Morton SC et al (2000) Meta-analysis of observational studies in epidemiology: a proposal for reporting. Meta-analysis Of Observational Studies in Epidemiology (MOOSE) group. JAMA 283:2008-2012

13. Katz S, Downs TD, Cash HR et al (1970) Progress in development of the index of ADL. Gerontologist 10:20-30

14. Sandvik H, Hunskaar S, Seim A et al (1993) Validation of a severity index in female urinary incontinence and its implementation in an epidemiological survey. J Epidemiol Community Health 47:497-499

15. Wells GA, Tugwell P, O'Connell D et al (2015) The NewcastleOttawa Scale (NOS) for assessing the quality of nonrandomized studies in meta-analyses. http://www.ohri.ca/programs/clinical_ epidemiology/oxford.asp. Accessed 1 June 2020

16. Luchini C, Stubbs B, Solmi M et al (2017) Assessing the quality of studies in meta-analysis: advantages and limitations of the Newcastle Ottawa Scale. World J Meta-Anal 5:1-48

17. Higgins JPT, Green S (2008) Cochrane handbook for systematic reviews. Hoboken, Chichester

18. Cohen J (1992) Statistical power analysis. Curr Dir Psychol Sci 1:98-101

19. Higgins JP, Altman DG, Gotzsche PC et al (2011) The Cochrane Collaboration's tool for assessing risk of bias in randomised trials. BMJ 343:d5928

20. Egger M, Davey Smith G, Schneider M et al (1997) Bias in metaanalysis detected by a simple, graphical test. BMJ 315:629-634

21. Duval S, Tweedie R (2000) A nonparametric "trim and fill" method of accounting for publication bias in meta-analysis. J Am Stat Assoc 95:89-98

22. Oh SJ, Ku JH (2007) Impact of stress urinary incontinence and overactive bladder on micturition patterns and health-related quality of life. Int Urogynecol J Pelvic Floor Dysfunct 18:65-71

23. Coyne KS, Sexton CC, Irwin DE et al (2008) The impact of overactive bladder, incontinence and other lower urinary tract symptoms on quality of life, work productivity, sexuality and emotional well-being in men and women: results from the EPIC study. BJU Int 101:1388-1395

24. Schimpf MO, Patel M, O'Sullivan DM et al (2009) Difference in quality of life in women with urge urinary incontinence compared to women with stress urinary incontinence. Int Urogynecol J Pelvic Floor Dysfunct 20:781-786

25. Tozun M, Ayranci U, Unsal A (2009) Prevalence of urinary incontinence among women and its impact on quality of life in a semirural area of Western Turkey. Gynecol Obstet Investig 67:241-249
26. Martínez Agulló E, Ruíz Cerdá JL, Gómez Pérez L et al (2010) Impact of urinary incontinence and overactive bladder syndrome on health-related quality of life of working middle-aged patients and institutionalized elderly patients. Act Urol Españ (Engl Ed) $34: 242-250$

27. Duggan P (2011) Urodynamic diagnoses and quality of life in women presenting for evaluation of urinary incontinence. Aust $\mathrm{N}$ Z J Obstet Gynaecol 51:416-420

28. Rannestad T, Skjeldestad FE (2011) Ferrans and Powers' Quality of life index applied in urinary incontinence research-a pilot study. Scand J Caring Sci 25:410-416

29. Aguilar-Navarro S, Navarrete-Reyes AP, Grados-Chavarria BH et al (2012) The severity of urinary incontinence decreases healthrelated quality of life among community-dwelling elderly. J Gerontol A Biol Sci Med Sci 67:1266-1271

30. de Mello Portella P, Feldner PC Jr, da Conceicao JC et al (2012) Prevalence of and quality of life related to anal incontinence in women with urinary incontinence and pelvic organ prolapse. Eur J Obstet Gynecol Reprod Biol 160:228-231

31. Horng SS, Huang N, Wu SI et al (2013) The epidemiology of urinary incontinence and it's influence on quality of life in Taiwanese middle-aged women. Neurourol Urodyn 32:371-376

32. Oliveira CD, Seleme M, Cansi PF et al (2013) Urinary incontinence in pregnant women and its relation with socio-demographic variables and quality of life. Rev Ass Méd Bras (Engl Ed) 59:460-466

33. Choi EP, Lam CL, Chin WY (2014) The health-related quality of life of Chinese patients with lower urinary tract symptoms in primary care. Qual Life Res 23:2723-2733

34. Tang DH, Colayco DC, Khalaf KM et al (2014) Impact of urinary incontinence on healthcare resource utilization, health-related quality of life and productivity in patients with overactive bladder. BJU Int 113:484-491

35. Lim R, Liong ML, Leong WS et al (2016) Effect of stress urinary incontinence on the sexual function of couples and the quality of life of patients. J Urol 196:153-158

36. Gascon MRP, Mellao MA, Mello SH et al (2018) The impact of urinary incontinence on the quality of life and on the sexuality of patients with HAM/TSP. Braz J Infect Dis 22:288-293

37. Lin YH, Chang SD, Hsieh WC et al (2018) Persistent stress urinary incontinence during pregnancy and one year after delivery; its prevalence, risk factors and impact on quality of life in Taiwanese women: an observational cohort study. Taiwan J Obstet Gynecol 57:340-345

38. De Nunzio C, Pastore AL, Lombardo R et al (2019) The EORTC quality of life questionnaire predicts early and long-term incontinence in patients treated with robotic assisted radical prostatectomy: analysis of a large single center cohort. Urol Oncol 37:1006-1013

39. Steibliene V, Aniuliene R, Aniulis P et al (2020) Affective symptoms and health-related quality of life among women with stress urinary incontinence: cross-sectional study. Neuropsychiatr Dis Treat 16:535-544

40. Xiao J, Caan BJ, Weltzien E et al (2018) Associations of preexisting co-morbidities with skeletal muscle mass and radiodensity in patients with non-metastatic colorectal cancer. J Cachexia Sarcopenia Muscle 9:654-663

41. Hawkins K, Pernarelli J, Ozminkowski RJ et al (2011) The prevalence of urinary incontinence and its burden on the quality of life among older adults with medicare supplement insurance. Qual Life Res 20:723-732

42. Balkarli A, Semiz M, Uslu AU et al (2016) An assessment of sleep disturbances and quality of life in primary Sjögren's syndrome and its relationship with urinary incontinence. Acta Med Mediter 32:917 
43. Goris S, Sungur G, Tasci S et al (2010) The effect of urinary incontinence and sexual dysfunction on the quality of life among women with multiple sclerosis. Pakistan J Med Sci 26:277-281.

44. Mallah F, Montazeri A, Ghanbari Z et al (2014) Effect of urinary incontinence on quality of life among Iranian women. J Fam Reprod Health 8:13

45. Jenkinson C, Coulter A, Wright L (1993) Short form 36 (SF36) health survey questionnaire: normative data for adults of working age. BMJ 306:1437-1440

46. Moore KN, Jensen L (2000) Testing of the Incontinence Impact Questionnaire (IIQ-7) with men after radical prostatectomy. J WOCN 27:304-312

47. Kwon BE, Kim GY, Son YJ et al (2010) Quality of life of women with urinary incontinence: a systematic literature review. Int Neurourol 14:133

48. Bartoli S, Aguzzi G, Tarricone R (2010) Impact on quality of life of urinary incontinence and overactive bladder: a systematic literature review. Urology 75:491-500

49. Norton P, Brubaker L (2006) Urinary incontinence in women. Lancet 367:57-67

50. Markland AD, Goode PS, Redden DT et al (2010) Prevalence of urinary incontinence in men: results from the national health and nutrition examination survey. J Urol 184:1022-1027

51. Offermans MP, Du Moulin MF, Hamers JP et al (2009) Prevalence of urinary incontinence and associated risk factors in nursing home residents: a systematic review. Neurourol Urodyn 28:288-294
52. Beele H, Smet S, Van Damme N et al (2018) Incontinence-associated dermatitis: pathogenesis, contributing factors, prevention and management options. Drugs Aging 35:1-10

53. Beeckman D, Campbell J, Campbell K et al (2015) Incontinenceassociated dermatitis: moving prevention forward. Wounds Int 9:21-25

54. Nygaard I, DeLancey J, Arnsdorf L et al (1990) Exercise and incontinence. Obs Gyn 75:848-851

55. Bogner HR, Gallo JJ, Swartz KL et al (2002) Anxiety disorders and disability secondary to urinary incontinence among adults over age 50. Int J Psychiatry Med 32:141-154

56. Shamliyan TA, Kane RL, Wyman J et al (2008) Systematic review: randomized, controlled trials of nonsurgical treatments for urinary incontinence in women. Ann Intern Med 148:459-473

57. Kinchen KS, Obenchain R, Swindle R (2005) Impact of duloxetine on quality of life for women with symptoms of urinary incontinence. Int Urogynecol J 16:337-344

58. Borello-France DF, Zyczynski HM, Downey PA et al (2006) Effect of pelvic-floor muscle exercise position on continence and quality-of-life outcomes in women with stress urinary incontinence. Phys Ther 86:974-986

Publisher's Note Springer Nature remains neutral with regard to jurisdictional claims in published maps and institutional affiliations. 